EPJ Web of Conferences 59, 19002 (2013)

DOI: $10.1051 /$ epjconf/20135919002

(C) Owned by the authors, published by EDP Sciences, 2013

\title{
Flyer acceleration experiments using high-power laser
}

\author{
T. Kadono ${ }^{1}$, T. Sakaiya ${ }^{2}$, Y. Hironaka ${ }^{1}$, H. Nagatomo ${ }^{1}$, T. Sano ${ }^{1}$, T. Watari ${ }^{3}$, \\ K. Otani ${ }^{4}$, T. Fujiwara ${ }^{1}$, T. Mochiyama ${ }^{1}$, S. Fujioka ${ }^{1}$, K. Shigemori ${ }^{1}$, H. Yabuta ${ }^{2}$, \\ T. Kondo ${ }^{2}$, M. Arakawa ${ }^{5}$, A.M. Nakamura ${ }^{5}$, K. Kurosawa ${ }^{7}$, S. Sugita ${ }^{6}$, \\ S. Ohno ${ }^{8}$ and T. Matsui ${ }^{8}$
}

1 Institute of Laser Engineering, Osaka University, 2-6 Yamadaoka, Suita, Osaka 565-0871, Japan

2 Department of Earth and Space Science, Osaka University, 1-1 Machikaneyama, Toyonaka, Osaka 560-0043, Japan

3 Hamamatsu Photonics K. K., 1820 Kurematsu, Nishi, Hamamatsu 431-1202, Japan

4 Institute for Chemical Research, Kyoto University, Gokasyou, Uji 611-0011, Japan

5 Department of Earth and Planetary Sciences, Kobe University, 1-1 Rokkodai, Nada, Kobe 657-8501, Japan

${ }^{6}$ Department of Complexity Science and Engineering, University of Tokyo,

5-1-5, Kashiwanoha, Kashiwa 277-8561, Japan

7 Institute of Space and Astronautical Science, Japan Aerospace Exploration Agency,

3-1-1, Yoshinodai, Sagamihara 252-5210, Japan

8 Planetary Exploration Research Center, Chiba Institute of Technology, 2-17-1 Tsudanuma, Narashino, Chiba 275-0016, Japan

\begin{abstract}
Flyer acceleration technique using high-power lasers has several advantages such as the achieved velocities higher than $10 \mathrm{~km} / \mathrm{s}$ and non-contamination to the products generated by impacts. In this study, we show that a high-power laser can achieve flyer velocities higher than $10 \mathrm{~km} / \mathrm{s}$ up to $60 \mathrm{~km} / \mathrm{s}$ using spherical projectiles with a diameter of $0.1-0.3 \mathrm{~mm}$. We discuss the projectile condition during the flight based on the results of numerical simulations.
\end{abstract}

\section{INTRODUCTION}

Impact experiments in planetary sciences have been mainly carried out with two-stage light-gas guns, with which macroscopic $(\gtrsim 0.1 \mathrm{~mm})$ projectiles are not easily accelerated to more than $10 \mathrm{~km} / \mathrm{s}$. Since, the average impact velocities of asteroids on Earth and other terrestrial planets during the final stages of planetary accretion are estimated to be higher than $10 \mathrm{~km} / \mathrm{s}$, the details of the effects by such impacts on the environments have not been understood well.

The irradiation of high-intensity lasers is a relatively familiar method to accelerate flyers to higher velocities than $10 \mathrm{~km} / \mathrm{s}$. High-power laser systems have been used to accelerate thin sheets to velocities above $10 \mathrm{~km} / \mathrm{s}$ to produce square-shaped shock waves without the pre-heating of targets [e.g., 1-11]. Since, for planetary interest, the impacts of projectiles with an aspect ratio of $\sim 1$ at velocities higher than $\sim 10 \mathrm{~km} / \mathrm{s}$ are highly desirable, we have developed a laser-gun, which can accelerate aluminium (Al) spheres to velocities higher than $10 \mathrm{~km} / \mathrm{s}$ [11-14]. Based on this flyer acceleration technique, the experiments of the impact vaporization and the investigation of the chemical compositions of the vapor

This is an Open Access article distributed under the terms of the Creative Commons Attribution License 2.0, which permits unrestricted use, distribution, and reproduction in any medium, provided the original work is properly cited. 


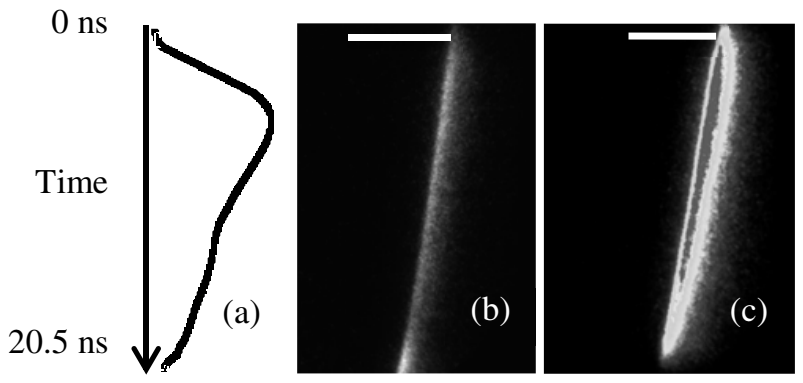

Figure 1. (a) Typical temporal profile of the laser. (b) and (c) Streak images of projectile acceleration process for (b) diamond and (c) Al. The laser irradiated from right. Self-luminous light from the ablation plasma was observed. The white bars indicate the projectile diameters, (b) 300 and (c) $250 \mu \mathrm{m}$, respectively.

have been carried out at high velocities $\gtrsim 10 \mathrm{~km} / \mathrm{s}[15,16]$. Also, the scaling laws of crater diameter and depth, silicate melt, size and the velocity distributions of ejecta [17], and recovering highly pressured minerals and organics at such high impact velocities, are now proceeding.

Here, first, we summarize the velocity of projectiles obtained by our laser-gun system. One important problem of the laser guns for spherical projectiles is the condition of the projectiles. To investigate the condition, we carry out some numerical simulations about the laser irradiation and acceleration of $\mathrm{Al}$ spheres, in which the laser conditions and the projectile velocity are consistent with the results of the experiments. Then, we discuss the thermodynamic conditions of projectiles based on the numerical results.

\section{EXPERIMENT AND RESULTS}

GEKKO XII-HIPER laser at Institute of Laser Engineering, Osaka University, was used to accelerate flyers. Laser wavelength, pulse width, and energy was $1.054 \mathrm{~nm}, \sim 10-20 \mathrm{~ns}$, and $\sim 0.8-5 \mathrm{~kJ}$, respectively. Typical temporal profile is shown in Fig. 1(a). Laser irradiated Al, glass, gold, and diamond spheres with a size of $0.1-0.3 \mathrm{~mm}$. A very thin surface of the projectiles vaporized and became highdensity and temperature plasma, which accelerated the projectiles. We observed the acceleration process using an x-ray streak camera. The position of the projectiles can be measured from the streak camera images as a function of time. Hence, the projecitle velocity is evaluated [11-14].

Figure 1 also shows two images of the acceleration process of (b) diamond and (c) Al projectiles obtained by the streak camera. Time is vertical and $20.5 \mathrm{~ns}$ from top to bottom. The projectiles were irradiated from right. The horizontal white bar in each figure is a scale of space indicating the projectile diameter, 300 and $250 \mu \mathrm{m}$, respectively, setting at the initial positions of the projectiles. We can observe self-luminous emission from ablation plasma on the right-hand side of the projectiles. We trace the positions of the laser irradiation surface as a function of time, and obtain the velocity of projectiles. The estimated final velocities in these shots are 10.5 and $12.2 \mathrm{~km} / \mathrm{s}$, respectively.

Figure 2 shows the final velocity $v$ of projectiles as a function of laser energy $E$ normalized by projectile mass $m_{\mathrm{p}}, 2 E / m_{\mathrm{p}}$. If a constant fraction $k$ of laser energy is transferred to the kinetic energy of projectiles, $v$ varies with $2 E / m_{\mathrm{p}}$ as $v=\left(2 \mathrm{kE} / \mathrm{m}_{\mathrm{p}}\right)^{1 / 2}$, indicated by straight lines for the cases of $k=1,0.1$, and $0.01 \%$. It can be seen that $v$ increases with $2 E / m_{\mathrm{p}}$, and most data exist between $k=0.1$ and $0.01 \%$, though there is some scatter.

\section{DISCUSSION}

Numerical simulations were performed to estimate the temperature, density, and velocity of the projectiles using a two-dimensional arbitrary-Lagrangian-Eulerian radiation hydrodynamic code for 


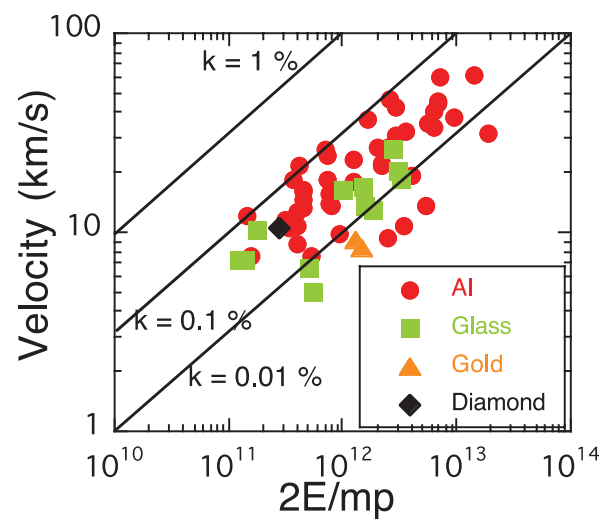

Figure 2. Projectile velocity as a function of laser energy over flyer mass. The transfer efficiency from laser to kinetic energy is $\sim 0.1-0.01 \%$.
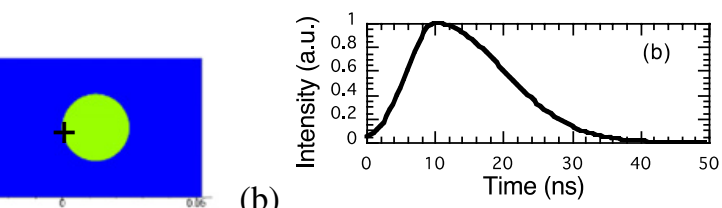

(a) $0 \mathrm{~ns}$

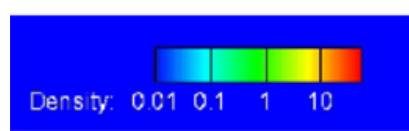

(1)
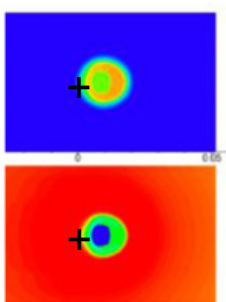

(c) $10 \mathrm{~ns}$

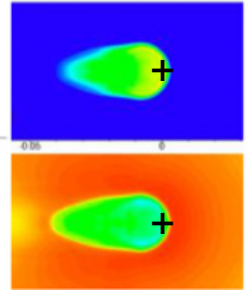

(d) $30 \mathrm{~ns}$

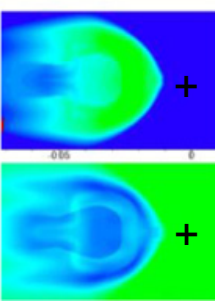

(e) $50 \mathrm{~ns}$

Figure 3. The results of numerical calculation. (a) Before the laser irradiation ( $0 \mathrm{~ns})$. The projectile is $\mathrm{Al}$ and the size is $300 \mu \mathrm{m}$. The position of the left hand side of the projectile is set at the origin and indicated by a cross. (b) Temporal profile of the laser. (c) - (e) The mass density in $\mathrm{g} / \mathrm{cm}^{3}$ (top) and the temperature in $\mathrm{eV}$ (bottom) contours as a function of time. The scale is the common with (a). (c) $10 \mathrm{~ns}$. Laser comes from right. The shock wave propagates into the projectile. (d) $30 \mathrm{~ns}$. The projectile moves to the left. (e) $50 \mathrm{~ns}$. The velocity of the projectile is $\sim 11 \mathrm{~km} / \mathrm{s}$, which is consistent with the experiments.

laser plasma, "PINOCO" (precision integrated implosion numerical observation code) [18]. Figure 3 shows a result of the numerical simulation for the case of an Al sphere with $300 \mu \mathrm{m}$ in diameter. (a) Before the laser irradiation. An $\mathrm{Al} 300 \mu \mathrm{m}$ sphere is set as the position of the left hand side of the projectile is the origin (indicated by a cross). The scale is common in all figures in Fig. 3. Laser irradiation starts at $0 \mathrm{~ns}$ from the right and the intensity increases with a Gaussian profile with $4 \mathrm{~ns}$ FWHM. Then, it peaks at $10 \mathrm{~ns}$ and decreases with another Gaussian with $10 \mathrm{~ns}$ FWHM (Fig. 3b).

Laser energy is $3 \mathrm{~kJ}$ and $60 \%$ is absorbed. The spatial profile of the laser is a Gaussian with $300 \mu \mathrm{m}$ FWHM. Figure 3 also shows the mass density in $\mathrm{g} / \mathrm{cm}^{3}$ (top) and the temperature in $\mathrm{eV}$ (bottom) contours, (c) $10 \mathrm{~ns}$ after the beginning of the laser irradiation; the shock wave propagates into the projectile, (d) $30 \mathrm{~ns}$ after the beginning of the irradiation; the projectile is accelerated to the left, and (e) $50 \mathrm{~ns}$ after the beginning of the irradiation; the temperature of the projectile decreases below $\sim$ a few 
thousand degrees while the mass density is an order of $\sim 1 \mathrm{~g} / \mathrm{cm}^{3}$. This suggests that the projectile does not vaporize. Since the resolution of this code at lower temperature than $\sim 1000 \mathrm{~K}$ is not enough, the accurate temperature (and density) at the later stages is not obtained and it is not clear whether the state of the Al projectile is solid or liquid at present. The velocity of the projectile is $\sim 11 \mathrm{~km} / \mathrm{s}$, which is consistent with the experiments. It should be noted that the projectile expands to lateral direction. This is probably because the projectile is not "solid" even at rather low temperature, since the simulation treats only gas and plasma, and recrystallization process is not taken into account.

\section{CONCLUSION}

Using a high-power laser, GEKKO XII-HIPER, we accelerate Al, glass, gold, and diamond spheres $0.1-0.3 \mathrm{~mm}$ in diameter to higher velocities than $10 \mathrm{~km} / \mathrm{s}$ up to $60 \mathrm{~km} / \mathrm{s}$. The numerical simulation indicates that the temperature of the projectile after the laser irradiation quickly decreases below thousand degree even at a density of $\sim 1 \mathrm{~g} / \mathrm{cm}^{3}$. This suggests that the projectile is not vapour before the impact to the targets.

\section{References}

[1] Obenschain, S. P., et al., Phys. Rev. Lett. 46, 1402-1405 (1981)

[2] Obenschain, S. P., et al., Phys. Rev. Lett. 50, 44-48 (1983)

[3] Fabbro, R., et al., J. Appl. Phys. 56, 3204-3208 (1984)

[4] Fabbro, R., et al., Phys. Fluids 28, 3414-3423 (1985)

[5] Fabbro, R., et al., Laser Part. Beams 4, 413-419 (1986)

[6] Faral, B., et al., Phys. Fluids B 2, 371-377 (1990)

[7] Cauble, M. D., et al. Phys. Rev. Lett. 70, 2102-2105 (1993)

[8] Tanaka, K. A., et al., Phys. Plasmas 7, 676-680 (2000)

[9] Kadono, T., et al., J. Appl. Phys. 88, 2943-2947 (2000)

[10] Kadono, T., et al., Laser Part. Beams 19, 623-630 (2001)

[11] Kadono, T., et al., Proc 17th APS Topical Conference on Shock Compression of Condensed Matter, pp. 847-850, AIP Conference Proceedings 1426 (2012)

[12] Kadono, T., et al., Proc Fifth International Conference on Inertial Fusion Sciences and Applications (IFSA2007), J. Phys. Conf. Ser. 112, 042014 (2008)

[13] Kadono, T., et al., Proc 16th APS Topical Conference on Shock Compression of Condensed Matter, pp. 875-877, AIP Conference Proceedings 1195 (2009)

[14] Kadono, T., et al., J. Geophys. Res. 115, E04003, doi:10.1029/2009JE003385 (2010)

[15] Kurosawa, K. et al., Geophys. Res. Lett. 37, L23203, doi: 10.1029/2010GL045330 (2010)

[16] Ohno, S., et al., Proc 17th APS Topical Conference on Shock Compression of Condensed Matter, pp. 851-854, AIP Conference Proceedings 1426 (2012)

[17] Takasawa, S., et al., Astrophys. J. Lett. 733, L39-L42 (2011)

[18] Nagatomo H., et al., Phys. Plasma 14, 056303, doi: 10.1063/1.2671124 (2007) 